AN. MED. INTERNA (Madrid) Vol. 18, N. ${ }^{\circ} 10$, pp. 510-516, 2001

\section{Tasas específicas de mortalidad por GRD y diagnóstico principal según la CIE-9-MC en un hospital de II nivel}

\author{
J. M. RAMOS RINCÓN, D. GARCÍA RUIPÉREZ*, F. ALIAGA MATAS*, \\ $\mathrm{M}^{\mathrm{a}} \mathrm{C}$. LOZANO CUTILLAS*, R. LLANOS LLANOS, F. HERRERO HUERTA \\ Servicio de Medicina Interna y *Unidad de Documentación Clínica. Hospital J.M. \\ Morales Meseguer. Murcia
}

RESUMEN

Introducción: La mortalidad hospitalaria es un indicador de la calidad asistencial, se puede evaluar según el diagnóstico principal al alta por éxitus o por los grupos relacionados con el diagnóstico (GRD).

Objetivos: Conocer y analizar la mortalidad y tasa de mortalidad (TM) hospitalaria en los ingresados en el Hospital Morales Meseguer (Murcia) de nivel II del INSALUD en el año 1999.

Metodología: Estudio del conjunto mínimo básico de datos al alta hospitalaria.

Resultados: La TM fue de 3,8\%, de los 508 fallecimientos analizados, el $56,7 \%$ correspondieron a varones. La TM fue similar en varones $(3,6 \%)$ y en mujeres $(3,9 \%)$. La mediana de edad de los fallecidos era de 77 años. El número de fallecimientos en menores de 80 años era mayor en varones $(n=197 ; 65 \%)$ que en mujeres $(n=102 ; 35 \%)(p=0,002)$. La suma de los éxitus de Medicina Interna y Cuidados Intensivos representa el 70\% de casos ocurridos en el hospital. La TM fue mayor en la Unidad de Cuidados Intensivos (10,5\%) seguido por Hematología $(6,9 \%)$ y Medicina Interna (6,1\%). Diez GRDs representa el 50\% de las causas de muertes, todos ellos son de tipo médico. El GRD más frecuente fue el 541. Trastornos respiratorios con complicación ( $\mathrm{n}=61, \mathrm{TM} 12,8 \%$ ), seguido por el GRD 533, Trastornos cerebrovasculares específicos excepto accidente isquémico transitorio $(\mathrm{n}=33$; TM $34,7 \%)$, GRD123 Infarto agudo de miocardio (IAM) con éxitus ( $\mathrm{n}=31$; TM 100\%), GRD 014 Otros trastornos del sistema nervioso $(n=27$; TM: $10,3 \%)$ y GRD 552 Trastornos del aparato digestivo excepto esofagitis, gastroenteritis y úlcera no complicada $(\mathrm{n}=27$; TM: 31,0$)$. El diagnóstico principal más frecuente fue la enfermedad cerebrovascular $(n=60$, TM 12,2\%), neumonía-bronconeumonía $(\mathrm{n}=52$, TM $10,9 \%)$, enfermedad pulmonar obstructiva crónica $(\mathrm{n}=36, \mathrm{TM} 7,1 \%)$, IAM $(\mathrm{n}=33, \mathrm{TM} 11,5 \%)$, y neoplasia del aparato digestivo $(\mathrm{n}=26$; TM $8,5 \%)$. La TM en las mujeres con IAM $(19,5 \%)$ fue superior a la los hombres $(7,7 \%)(\mathrm{p}=0,005)$, la TM en las mujeres con hernia abdominal $(4,3 \%)$ fue superior a la de los hombres $(0,6 \%)(p=0,01)$, y La TM de la pielonefritis e infección urinaria en los varones $(4,9 \%)$ fue mayor a la de las mujeres $(0 \%)(\mathrm{p}=0,008)$.

Conclusión: La mortalidad y TM por GRD y diagnóstico principal es un indicador que permite conocer y monitorizar la calidad de la asistencia sanitaria.

PALABRAS CLAVE: Mortalidad hospitalaria. Grupos relacionados con el diagnóstico. Diagnóstico principal. Control de calidad.
MORTALITY RATE BY DRG AND BY THE PRINCIPAL DIAGNOSIS ACCORDING THE ICD-9-CM IN A II LEVEL HOSPITAL OF INSA LUD OF MURCIA DURING 1999

\section{ABSTRACT}

Introduction: The inhospital mortality is a marker of health care quality. It can be evaluated according by the principal diagnosis of dise ase or by Diagnosis Related Groups (DRG).

Objectives: know and to analyze the inhospital mortality and morta lity rate (MR) in the patients admitted in the Hospital Morales Meseguer (Murcia) of II level of the INSALUD during 1999.

Methodology: Study of the minimum basic group of data into the hospital.

Results: The MR was $3.8 \%$ of 508 deaths analyzes, $56.7 \%$ corres ponded a male. The MR was equal in male (3.6\%) and females (3.9\%). The medium of age into deaths was 77 years. The numbers of deaths inpatients under 80 years was higher in males $(n=197 ; 65 \%)$ than in lower in female $(n=102 ; 35 \%)(p=0.002)$. The addition of deaths of Internal Medicine and Intensive Care Unit represent a $70 \%$ of cases of deaths occurred in the hospital. The MR was higher in Intensive Care Unit (10.5\%) followed by Hematology (6.9\%) and Internal Medicine (6.1\%). Ten DRG represents of $50 \%$ of the causes of deaths, and all of them were $D R G$ of medical categories. The DRG more frequents was 541, Respira tory disorders with complication ( $n=61, M R 12.8 \%$ ), followed by DRG 533, Specific cerebrovascular disorders without Transit isquemic acci dent ( $n=33 ; M R 34.7 \%), D R G 123$ Acute myocardical infarction (AMI) with death (n=31; MR100\%), DRG 014 Others disorders of nervous sys tem (n= 27; TM: 10.3\%) y DRG 552 Disorders of digestive tract without esophagitis, gastroenteritis and not complicated ulcer $(n=27 ;$ MR: 31.0$)$. The main diagnostic more frequent was cerebrovascular diseases $(n=60$, MR12.2\%), pneumonia-bronchopneumonia $(n=52$, MR 10.9\%), chronic obstructive lung disease $(n=36, M R 7.1 \%), A M I(n=33, M R 11.5 \%)$, and neoplasia of digestive tract $(n=26 ; M R 8.5 \%)$. The MR of woman with AMI $(19.5 \%)$ was higher than man $(7.7 \%)(p=0.005)$, the MR in woman with abdominal hernia (4.3\%) was higher than man $(0.6 \%)(p=0.01)$, and MR of pyelonephritis and urinary infection in man (4.9\%) was hig her than woman $(0 \%)(p=0.008)$.

Conclusions: The mortality and MR by DRG and main diagnostic is an indicator than permit to know and monitoring the quality of health care.

KEY WORDS: Inhospital mortality. Diagnosis realated groups. Main diagnostic. Quality control.

Ramos Rincón JM, García Ruipérez D, Aliaga Matas F, Lozano Cutillas MC, Llanos Llanos R, Herrero Huerta F. Tasas específicas de morta lidad por GRD y diagnóstico principal según la CIE-9-MC en un hospital de II nivel. An Med Interna (Madrid) 2001; 18: $510-516$.

Trabajo aceptado: 14 de Mayo de 2001

Correspondencia: José M. Ramos Rincón. Servicio de Medicina Interna. Hospital General Universitario. Camí de L'Almazara, 11. 03303 Elche. E-mail: jramosr@seimc.org. 


\section{INTRODUCCIÓN}

La medida cuantitativa de la mortalidad es el más común y más antiguo indicador sanitario que se conoce. La mortalidad hospitalaria debe ser interpretada con precaución al verse influenciada por diferentes factores como patología atendida, la estructura poblacional, el régimen económico del centro y la accesibilidad al centro sanitario (1). Constituye un instrumento muy útil en la investigación clínica y epidemiológica, en la evaluación de los programas de garantía de la calidad y en el conocimiento de los problemas de salud de la población atendida por el hospital $(2,3)$. La evaluación de la mortalidad se puede realizar mediante bases de datos clínicas o administrativas. Las bases de datos administrativas son las más utilizadas para evaluar la mortalidad hospitalaria (1,3-5), si bien su uso tiene limitaciones ya que no incorporan la gravedad del proceso para predecir la mortalidad y miden resultados para los que no fueron diseñadas (1,3). La base administrativa más comúnmente utilizada es la del conjunto mínimo básico de datos (CMBD) y el estudio de la mortalidad hospitalaria se realiza en función del diagnóstico principal según la Clasificación Internacional de Enfermedades (CIE) $(1,4,5)$ y en menor medida mediante el análisis de los Grupos Relacionados con el Diagnóstico (GRDs) (1,3).

En el estudio de la mortalidad hospitalaria hay autores que prefieren analizan la mortalidad ocurrida durante un periodo de tiempo tras el ingreso hospitalario, para evitar el sesgo de una potencial política de altas domiciliarias para enfermos terminales (6). Otros investigadores limitan el estudio de la mortalidad a menores de 80 años para evitar el sesgo de la alta complejidad de estos pacientes sobre los que se adoptan actitudes menos agresivas y más expectantes (3). Mientras que ciertos autores seleccionan la mortalidad en los ingresados de más de 24 horas para así limitar el estudio de la mortalidad exclusivamente al ámbito hospitalario (4).

Se dispone de diferentes estudios de mortalidad hospitalaria realizados en hospitales de tercer nivel y segundo nivel. La mayoría de los trabajos presentan su información con datos absolutos y son pocos los autores que estudian y analizan la mortalidad en forma de tasa $(1,3)$. El objetivo de este trabajo es presentar la mortalidad y la tasa de mortalidad (TM) de los pacientes ingresados en un hospital de II nivel del INSALUD de Murcia durante el año 1999.

\section{MATERIAL Y MÉTODO}

El Hospital Morales Meseguer es un hospital del INSALUD de Murcia con 340 camas, que tiene adscrita la población de la Vega Media del Segura y parte de la ciudad de Murcia con 230.000 habitantes. El hospital no dispone de los servicios de Pediatría, Obstetricia, y Nefrología.

La información se recogió del informe clínico de alta y/o historia clínica para los datos clínicos, cumplimentándose el CMBD al alta hospitalaria, codificado mediante la Clasificación Internacional de Enfermedades $9^{a}$ edición modificación clínica (CIE-9-MC). Con dicha información se obtuvieron y procesaron los grupos de diagnóstico relacionado (GRD, versión AP-GRD 10.0) mediante el programa informático "Estación Clínica Analizador CASEMIX AP 14.1 GRD” 4.0. Los datos administrativos se recogieron al ingreso y al alta.
Las variables estudiadas para cada episodio de hospitalización han sido: sexo, edad, servicio de alta, diagnóstico principal, y GRD motivo del alta. Dado el alto número de diagnósticos de la CIE-9-MC, se agruparon los enfermos en patologías similares desde un punto de vista clínico. Se ha calculado la TM de cada categoría, definida como el cociente entre los fallecidos por una causa determinada y las altas hospitalarias por esa misma causa en el periodo de estudio, multiplicado por 1000. En el cálculo de la TM para la Unidad de Cuidados Intensivos en el denominador del cociente se empleó el número de ingresos existentes en la Unidad, en vez del número de altas de la Unidad recogidas en la Unidad de Documentación Clínica, ya que únicamente se contabilizan las altas por traslado y éxitus.

Las variables estudiadas para cada episodio de hospitalización han sido: sexo, edad, servicio de alta, diagnóstico principal, y GRD resultante al alta. A la estimación puntual se le ha calculado el intervalo de confianza del 95\% (IC 95\%). Para la magnitud de la diferencia entre dos categorías se ha empleado el riesgo relativo (RR) con el IC 95\%. En el estudio comparativo de variables cualitativas se ha empleado la prueba de chi cuadrado de Pearson y la prueba de Fisher. Se ha considerado como nivel de significación estadística un error alfa $<0,05$. La base de datos fue gestionada mediante el programa Excel 7.0 y el análisis estadístico fue procesado mediante el programa Epi Info v 6.04 y SPSS 8.0.

\section{RESULTADOS}

Durante el año de estudio 1999 se han producido en el centro hospitalario un total de 103.010 estancias y una media de 7,09 días de estancia. El total de ingreso y altas hospitalarias fue de 14.521 y 14.493, respectivamente (según la estadística del hospital), de ellas 576 correspondían a éxitus $(3,9 \%)$. El número de altas de las que se disponen informe codificado la Unidad de Documentación Clínica fue de 13.516, y de estas se dispone se ha codificado el éxitus en 508 ocasiones $(3,7 \%$, IC $95 \% 3,4-4,1)$. Se tha trabajado con los datos existentes en la Unidad de Documentación Clínica.

De los 508 fallecimientos analizados, 278 (56,7\%) correspondieron a varones y $230(43,3 \%)$ a mujeres. La TM fue similar en ambos grupos (3,6 y 3,9\%, respectivamente). La edad media de los fallecidos era de 75,6 años y la mediana de 77 años (recorrido intercuartílico 70-84). Sólo el 7,7\% de los fallecimientos correspondían a menores de 60 años, el 52\% ocurrió en sujetos entre los 60 y 79 años y el 40,2\% en mayores de 79 años (p<0,001). El número de fallecimientos en menores de 80 años era mayor en varones $(n=197 ; 65 \%)$ que en mujeres $(n=102 ; 35 \%)$, si bien el porcentaje se invertía en fallecidos con edad igual o superior a 80 años $(37,7$ y $62,3 \%$, respectivamente $)(p=0,002)$. Ciento veintinueve $(25,4 \%)$ fallecieron en las primeras cuarenta y ocho horas.

Por servicios asistenciales los que mayor muertes acumularon fueron los de Medicina Interna y Cuidados Intensivos, ya que la suma de los éxitus de ambos representa el $70 \%$ de casos ocurridos en el hospital. La TM fue mayor en la Unidad de Cuidados Intensivos (10,5\%) seguido por Hematología $(6,9 \%)$ y Medicina Interna $(6,1 \%)$ (Tabla I). En el análisis del número de fallecimientos por sexo en cada servicio destacaba: a) en el Servicio de Cirugía General un porcentaje de hombres y mujeres al alta $(56,4$ y $43,6 \%)$ distinto al de éxitus $(33,4$ y $63,6 \%$, respecti- 
TABLA I

NÚM ERO DE FALLECIM IENTOS Y TASA DE M ORTALIDAD (TM ) POR SERVICIO

\begin{tabular}{|c|c|c|c|c|c|c|c|c|c|c|}
\hline \multirow[t]{2}{*}{ Servicio } & \multicolumn{2}{|c|}{ № de muertes } & \multicolumn{2}{|c|}{ TM \# } & \multicolumn{3}{|c|}{ Hombres } & \multicolumn{2}{|c|}{ Mujeres } & \multirow[b]{2}{*}{ TM } \\
\hline & & & & IC $95 \%$ & № & & TM & № & & \\
\hline M edicina Interna & 246 & $(48,1)$ & 6,1 & $5,4-6,9$ & 136 & $(55,3)$ & 6,3 & 110 & $(44,7)$ & 6,0 \\
\hline Unidad de Cuidados Intensivos & 123 & $(21,2)$ & 10,5 & $8,8-12,5$ & 73 & $(50)$ & & 50 & $(41,9)$ & \\
\hline Cirugía general & 44 & $(8,7)$ & 1,5 & $1,1-2,0$ & 16 & $(33,4)$ & $0,9 *$ & 28 & $(63,6)$ & $2,2 *$ \\
\hline Neurología & 27 & $(5,3)$ & 4,7 & $3,2-6,8$ & 13 & $(48,2)$ & 4,2 & 14 & $(51,8)$ & 5,2 \\
\hline Neumología & 17 & $(3,3)$ & 2,9 & $1,7-4,7$ & 11 & $(64,7)$ & 2,6 & 6 & $(35,3)$ & 3,5 \\
\hline Traumatología & 16 & $(3,1)$ & 1,1 & $0,6-1,8$ & 3 & $(18,7)$ & $0,6 * *$ & 13 & $(81,3)$ & $1,6 * *$ \\
\hline Digestivo & 14 & $(2,7)$ & 3,1 & $1,7-5,2$ & 11 & $(78,6)$ & 3,9 & 3 & $(21,4)$ & 0,7 \\
\hline Cardiología & 8 & $(1,6)$ & 0,6 & $0,3-1,2$ & 4 & $(50)$ & 0,4 & 4 & $(50)$ & 0,7 \\
\hline Hematología & 7 & $(1,3)$ & 6,9 & $3,1-14,2$ & 5 & $(71,4)$ & 10,8 & 2 & $(28,6)$ & 3,6 \\
\hline Urología & 5 & $(0,9)$ & 0,8 & $0,3-2,0$ & 5 & $(100)$ & 1,0 & 0 & (0) & 0 \\
\hline Dermatología & 1 & $(0,2)$ & 0,6 & $0,03-3,9$ & 1 & $(100)$ & 1,0 & 0 & (0) & 0 \\
\hline Total & 508 & & & & & & & & & \\
\hline
\end{tabular}

№: número; IC 95\%: intervalo de confianza del 95\%. Entre paréntesis porcentajes. \#Tasa de mortalidad por servicio: № muertes en el servicio / № altas en el servicio. Excepto en la Unidad de Cuidados Intensivos donde se ha contabilizado como denominador el $\mathrm{N}$ ㅇ de ingresos en la unidad.

$* p=0,01 ; * * p=0,02$

\section{TABLA II}

GRUPOS RELACIONADOS CON EL DIAGNÓ STICO (GRD) MÁS FRECUENTES DE LOS ÉXITUS CON SUS TASAS DE MORTALIDAD (TM)

\begin{tabular}{|c|c|c|c|c|c|c|}
\hline$\overline{G R D}$ & Descripción & № muertes & $\%$ & IC $95 \%$ & $\begin{array}{c}\mathrm{TM} \\
\%\end{array}$ & IC $95 \%$ \\
\hline 541 & Trastornos respiratorios excepto infecciones, bronquitis, asma con cc mayor & 61 & 12 & $9,3-15,2$ & 12,8 & $10,0-16,2$ \\
\hline 533 & O tros trastornos del SN excepto AIT, convulsiones y cefalea con cc mayor & 33 & 6,5 & $4,6-9,1$ & 34,7 & $25,4-45,3$ \\
\hline 123 & Trastornos circulatorios con IAM, éxitus & 31 & 6,1 & $4,2-8,6$ & 100 & $86,3-100$ \\
\hline 014 & Trastornos cerebrovasculares específicos excepto AIT & 27 & 5,3 & $3,6-7,7$ & 10,3 & $7,0-14,7$ \\
\hline 552 & Trastornos aparato digestivo excepto esofagitis, gastroenteritis y úlcera no complicada & & & & & \\
\hline 55 & $\begin{array}{l}\text { con cc mayor } \\
\text { Procedimiento mayor sobre estómago, esófago, duodeno, intestino delgado y grueso }\end{array}$ & 27 & 5,3 & $3,6-7,7$ & 31,0 & $21,8-41,9$ \\
\hline & con cc mayor & 24 & 4,7 & $3,1-7,0$ & 100 & $82,8-100$ \\
\hline 544 & ICC y arritmia cardiaca con cc mayor & 21 & 4,1 & $2,6-6,3$ & 3,9 & $2,5-5,9$ \\
\hline 127 & Insuficiencia cardiaca y shock & 14 & 2,8 & $1,6-4,7$ & 5,3 & $3,1-9,0$ \\
\hline 475 & Diagnóstico del sistema respiratorio con ventilación asistida & 13 & 2,6 & $1,4-4,4$ & 54,1 & $33,2-73,8$ \\
\hline 082 & Neoplasias respiratorias & 13 & 2,6 & $1,4-4,4$ & 16,2 & $9,2-26,5$ \\
\hline 483 & Traqueostomía, excepto por trastornos de boca, laringe o faringe & 12 & 2,4 & $1,3-4,2$ & 66,6 & $41,1-85,6$ \\
\hline 557 & Trastornos hepatobiliares y de páncreas con cc mayor & 12 & 2,4 & $1,3-4,2$ & 11,4 & $6,3-19,5$ \\
\hline 584 & Septicemia con cc mayor & 11 & 2,2 & $1,1-3,9$ & 45,8 & $26,1-66,8$ \\
\hline 553 & Procedimientos sobre aparato digestivo excepto hernia y procedir & & & & & \\
\hline & estómago o intestino, con cc mayor & 10 & 2,0 & $1,0-3,7$ & 17,8 & $9,3-30,8$ \\
\hline 558 & Procedimiento sobre sistema musculoesquelético mayor excepto articulación mayor & & & & & \\
\hline & bilateral o múltiple con cc mayor & 10 & 2,0 & $1,0-3,7$ & 16,4 & $8,5-28,5$ \\
\hline 543 & Trastornos circulatorios excepto IAM, endocarditis, ICC y arritmia con cc mayor & 8 & 1,6 & $0,7-3,2$ & 11,9 & $5,6-22,7$ \\
\hline 542 & Bronquitis y asma con cc mayor & 7 & 1,4 & $0,6-3,9$ & 5,4 & $2,3-11,2$ \\
\hline 089 & Neumonía simple y pleuritis edad > 17 con complicaciones & 6 & 1,2 & $0,5-2,7$ & 4,0 & $1,6-9,0$ \\
\hline 203 & Neoplasia maligna del sistema hepatobiliar o de páncreas & 6 & 1,2 & $0,5-2,7$ & 10,9 & $4,5-22,9$ \\
\hline 078 & Embolismo pulmonar & 5 & 1,0 & $0,4-2,4$ & 12,5 & $4,7-27$ \\
\hline 18 & 0 tros diagnósticos de aparato digestivo edad > 17 con complicaciones & 5 & 1,0 & $0,4-2,4$ & 17,9 & $6,8-37,6$ \\
\hline 540 & Infecciones e inflamaciones respiratorias con cc mayor & 5 & 1,0 & $0,4-2,4$ & 20,0 & $7,6-41,3$ \\
\hline & Otros & 147 & & & & \\
\hline
\end{tabular}

TM : tasa de mortalidad; \% : porcentaje; IC 95\% : intervalo de confianza del 95\% ; SN: sistema nervioso; IAM : infarto agudo de miocardio; ICC: insuficiencia cardiaca congestiva; cc: complicaciones.

vamente; $\mathrm{p}=0,01$ ), es decir la mortalidad era mayor en las mujeres que en los hombres ingresados en dicho Servicio, y b) igualmente, en el Servicio de Traumatología fallecieron más mujeres
$(81,3 \%)$ respecto al porcentaje de mujeres dadas de alta $(53,4 \%)(\mathrm{p}=0,02)$.

La tabla II recoge los GRD con mayor número de muertes. 
Diez GRD representaban el $50 \%$ de las causas de muertes, todos ellos fueron de tipo médico. El GRD más frecuentes fue el 541, Trastornos respiratorios con complicación, seguido por el GRD 533, Trastornos cerebrovasculares específicos excepto accidente isquémico transitorio (AIT), GRD123 Infarto agudo de miocardio (IAM) con éxitus, GRD 014 Otros trastornos del sistema nervioso y GRD 552 Trastornos del aparato digestivo excepto esofagitis, gastroenteritis y úlcera no complicada. La TM fue del $100 \%$ en el GRD 123 (IAM con éxitus), como es lógico por figurar el éxitus en la definición del GRD, y en el GRD 582 (procedimiento mayor sobre estómago, esófago, duodeno, intestino delgado y grueso con complicación mayor). Hasta 18 GRD tenían una TM superior al $10 \%$ (Tabla II). Agrupando los GRD, el conjunto más frecuente fue el secundario a IAM e ICC (GRD: 123,127, 543, $544)$ con 74 casos $(14,6 \%)$, seguido de las infecciones y trastornos respiratorios, bronquitis y asma con complicaciones (GRD: 540, 541, 542) con 73 casos $(14,4 \%)$ seguido de los Trastornos cerebrovasculares (GRDs: 014, 533) $(\mathrm{n}=60$; $11,8 \%$ ) y Trastornos del aparato digestivo y hepatobiliar y sus neoplasias (GRDs: 552,553,557,203,188) con 60 casos ambos $(11,8 \%)$.

La tabla III muestra el diagnóstico principal de los éxitus. El diagnóstico principal más frecuente fue la enfermedad cerebrovascular, neumonía-bronconeumonía (incluida por aspiración), enfermedad pulmonar obstructiva crónica, IAM, neoplasia del aparato digestivo como entidades médicas; la insuficiencia vascular del intestino como enfermedad médico- quirúrgica y la fractura de fémur como situación traumatológica. Destaca la TM del 100\% del diagnóstico principal shock y shock cardiogénico, seguido de la insuficiencia vascular del intestino (68\%) y la neoplasia secundaria de aparatos respiratorio y digestivo $(24,5 \%)$, tromboembolismo de pulmón $(17,8 \%)$, neoplasia de hígado, vesícula, tracto biliar y páncreas $(14,5 \%)$, obstrucción intestinal sin hernia $(13,5 \%)$, la neoplasia de bronquio y pulmón $(12,6 \%)$, enfermedad cerebrovascular $(12,2 \%)$ e IAM $(11,5 \%)$.

En los varones el principal diagnóstico de defunción fue la neumonía $(n=30 ; 10,8 \%)$, seguido de EPOC y procesos afines $(\mathrm{n}=28 ; 10 \%)$, enfermedad cerebrovascular $(\mathrm{n}=27 ; 9,7 \%)$, IAM $(\mathrm{n}=15 ; 5,4 \%)$ y neoplasia del tracto digestivo $(\mathrm{n}=14 ; 3,9 \%)$. En las mujeres las causas de defunción más frecuentes son la enfermedad cerebrovascular $(n=33 ; 14,3 \%)$, la ICC $(n=22$; $9,6 \%)$, IAM ( $\mathrm{n}=18 ; 7,8 \%)$ y la neumonía y bronconeumonía $(\mathrm{n}=17 ; 7,4 \%)$. La distribución de la mortalidad por grupos de edad y sexo se recoge en la tabla IV. Las diferentes TM de los diagnósticos principales de los éxitus entre los hombres y mujeres se resumen en la tabla $\mathrm{V}$ y destaca: a) una mayor TM por IAM de las mujeres (19,5\% IC 95\%: 12,3-14,4\%) respecto a los hombres $(7,7 \%$, IC $95 \%: 4,5-12,5)(\mathrm{p}=0,005)$ con un riesgo relativo de 2,6 veces superior para las mujeres (IC 95\%: 1,3-4,8); b) mayor TM en las mujeres con hernia abdominal $(4,3 \%$ IC, $95 \%$ : $1,8-9,6)$ respecto a los hombres $(0,6 \%$ IC $95 \%$ : $0,1-2,6 \%)(\mathrm{p}=0,01)$ con un riesgo relativo para las mujeres de 6,9 (IC 95\%: 1,4-3,8) superior a los varones; y c) mayor TM de la pielonefritis e infección urinaria en los varo-

TABLA III

DIAGNÓSTICO PRINCIPAL DE LOS ÉXITUS MÁS FRECUENTE CON SU TASA DE M ORTALIDAD (TM)

\begin{tabular}{|c|c|c|c|c|c|}
\hline Código CIE-9-MC & Diagnóstico principal & № muertes & $\%$ muertes & $\begin{array}{c}\mathrm{TM} \\
\%\end{array}$ & IC $95 \%$ \\
\hline $430-435,9$ & Enfermedad cerebrovascular & 60 & 11,8 & 12,2 & $9,5-15,5$ \\
\hline $482-486,507,0$ & Neumonía, bronconeumonía y neumonía por aspiración & 52 & 10,2 & 10,9 & $8,3-14,1$ \\
\hline $491-496$ & EPOC, BCO, enfisema y asma & 36 & 7,1 & 4,3 & $3,0-5,9$ \\
\hline 410 & Infarto agudo de miocardio & 33 & 6,5 & 11,5 & $8,1-15,8$ \\
\hline 428 & Insuficiencia cardiaca & 26 & 5,1 & 8,5 & $5,7-12,3$ \\
\hline $150-156$ & Neoplasia esófago, estomago, colon y recto & 23 & 4,5 & 10,8 & $7,1-15,9$ \\
\hline 557 & Insuficiencia vascular del intestino & 17 & 3,3 & 68 & $46,4-84,3$ \\
\hline $820.03-820,8$ & Fractura de cuello de fémur y perrocanterea & 16 & 3,1 & 6,5 & $3,8-10,5$ \\
\hline $531,532,578,9$ & Ulcera gastroduodenal y hemorragia gastrointestinal & 13 & 2,6 & 6,3 & $2,6-13,8$ \\
\hline $162.3-162,9$ & Neoplasia de bronquio y pulmón & 12 & 2,4 & 12,6 & $7,0-21,4$ \\
\hline $197.0-197,7$ & Neoplasia maligna secundaria de los aparatos respiratorio y digestivo & 12 & 2,4 & 24,5 & $13,8-39,2$ \\
\hline $785.5-785,59$ & Shock y shock cardiogénico & 12 & 2,4 & 100 & $69,8-100$ \\
\hline $155-157,9$ & Neoplasia de hígado, vesícula, tracto biliar y páncreas & 11 & 2,1 & 14,5 & $7,8-24,8$ \\
\hline $426,0,427$ & Bloqueo auriculoventricular completo y disritmia cardiaca & 11 & 2,1 & 3,2 & $1,7-5,8$ \\
\hline 560 & O bstrucción intestinal sin hernia & 11 & 2,1 & 13,5 & $7,3-23,4$ \\
\hline 415.19 & Tromboembolismo de pulmón & 10 & 2,0 & 17,8 & $9,3-30,8$ \\
\hline 038 & Septicemia & 9 & 1,8 & 28,1 & $14,4-47$ \\
\hline 466 & Bronquitis aguda & 9 & 1,8 & 3,4 & $1,6-6,5$ \\
\hline $574-577$ & Colelitiasis, trastornos de la vesícula biliar y pancreatitis & 9 & 1,8 & 1,5 & $0,7-3,0$ \\
\hline 185,188 & Neoplasia de próstata y vejiga & 8 & 1,6 & 5,4 & $1,2-8,3$ \\
\hline $550-553$ & Hernia abdominal & 8 & 1,6 & 1,8 & $0,8-3,6$ \\
\hline 571 & Enfermedad hepática crónica y cirrosis & 5 & 0,9 & 3,9 & $1,5-9,4$ \\
\hline 250 & Diabetes mellitus & 5 & 0,9 & 2,3 & $0,8-5,6$ \\
\hline \multirow[t]{2}{*}{$590.1,599.0$} & Pielonefritis aguda e infección urinaria, sitio no especificado & 5 & 0,9 & 1,9 & $0,7-4,7$ \\
\hline & 0 tros & 95 & & & \\
\hline
\end{tabular}

TM : tasa de mortalidad; \% : porcentaje; IC 95\% : intervalo de confianza del $95 \%$; 
TABLA IV

DIAGNÓSTICO PRINCIPAL DE ÉXITUS POR SEXO

\begin{tabular}{|c|c|c|c|}
\hline \multicolumn{2}{|l|}{ Hombres } & \multicolumn{2}{|c|}{ M ujeres } \\
\hline Diagnóstico principal & № éxitus & Diagnóstico principal & № de éxitus \\
\hline $\begin{array}{l}\text { M enores de } 60 \text { años } \\
\text { Infección por VIH } \\
\text { Patología pancreáticobiliar } \\
\text { Neoplasia de pulmón } \\
\text { Cirrosis } \\
\text { Neumonía } \\
\text { O tros } \\
\text { Total }\end{array}$ & $\begin{array}{l}3(11,3) \\
2(5,9) \\
2(5,9) \\
2(5,9) \\
22 \\
34\end{array}$ & $\begin{array}{l}\text { Neoplasia maligna secundaria } \\
\text { Isquemia intestinal } \\
\text { O bstrucción intestinal } \\
\text { Factura de fémur } \\
\text { O tros } \\
\text { Total }\end{array}$ & $\begin{array}{l}2(40) \\
1(20) \\
1(20) \\
1(20) \\
0 \\
5\end{array}$ \\
\hline $\begin{array}{l}\text { Edad } 60-79 \text { años } \\
\text { Accidente cerebrovascular } \\
\text { EPOC, BCO, enfisema y asma } \\
\text { Neumonía } \\
\text { Neoplasia de esófago, estómago, colon y recto } \\
\text { Embolismo de pulmón } \\
\text { Insuficiencia cardiaca } \\
\text { O tros } \\
\text { Total }\end{array}$ & $\begin{array}{l}17(10,2) \\
17(10,2) \\
13(7,8) \\
7(4,2) \\
7(4,2) \\
7(4,2) \\
99 \\
167\end{array}$ & $\begin{array}{l}\text { Infarto agudo de miocardio } \\
\text { Accidente cerebrovascular } \\
\text { Insuficiencia cardiaca } \\
\text { Neumonía }\end{array}$ & $\begin{array}{c}13(13,4) \\
12(12,4) \\
7(7,2) \\
6(6,2)\end{array}$ \\
\hline $\begin{array}{l}\text { M ayores de } 79 \text { años } \\
\text { Neumonía } \\
\text { Accidente cerebrovascular } \\
\text { EPOC, BCO, enfisema y asma } \\
\text { Neoplasia de esófago, estómago, colon y recto } \\
\text { Infarto agudo de miocardio } \\
\text { Otros } \\
\text { Total }\end{array}$ & $\begin{array}{l}14(18,2) \\
10(12,3) \\
10(12,3) \\
7(9,1) \\
7(9,1) \\
29 \\
77\end{array}$ & $\begin{array}{l}\text { Accidente cerebrovascular } \\
\text { Neumonía } \\
\text { Insuficiencia cardiaca } \\
\text { Isquemia intestinal } \\
\text { Trastornos de la conducción } \\
\text { Otros } \\
\text { Total }\end{array}$ & $\begin{array}{l}21(16,5) \\
14(11) \\
10(7,9) \\
8(6,3) \\
5(3,9) \\
69 \quad 127\end{array}$ \\
\hline
\end{tabular}

Entre paréntesis porcentajes. EPO C: enfermedad pulmonar obstructiva crónica. BCO : broncopatía crónica obstructiva

nes $(4,9 \%$, IC $95 \%: 1,8-11,6)$ respecto a las mujeres $(0 \%$ IC 95\%: $0-2,9)(\mathrm{p}=0,008)$.

\section{DISCUSIÓN}

La mortalidad hospitalaria como fuente de datos de mortalidad debe ser interpretado con precaución, si bien puede proporcionar datos de interés sobre el funcionamiento del propio hospital y supone un indicador de calidad asistencial (7).

La mortalidad general adecuada del hospital debería estar en menos del 4\% (7), en este hospital fue del 4,2\% de los casos. Si nos limitáramos a estudiar la mortalidad en los que llevaban más de 48 horas según Rodríguez Navarro y cols. (7) el porcentaje sería menor. En diferentes estudios de hospitales nacionales la mortalidad oscila entre 2,95\%-5,78\% $(1,4,5,7,8)$. En los hospitales de tercer nivel la mortalidad es mayor, en torno al 5,5\% $(7,8)$, en los de segundo nivel alrededor del 3-4\% (5) y en los de primer nivel, generalmente, menor al 3\% (4). Con respecto a la edad, destaca la mayor mortalidad en los pacientes mayores de 60 años, como sucede en la mayoría de los estudios observados $(4,5,7)$.

La mortalidad es mayor en varones que en mujeres $(1,3,4,7$,$) , si bien la TM no varía con el sexo (8,9)$ como sucede en este caso; no obstante en el estudio de García y cols. (3) y De Escante Yangüela y cols. (11) la TM fue significativamente superior en los varones. Entre los resultados de este trabajo destaca un mayor número de fallecimientos en los hombres de menos de 80 años y en las mujeres a partir de esa edad, esto se puede atribuir a la mayor longevidad de las mujeres en nuestro entorno (11). Otro dato a destacar es la TM significativamente mayor en las mujeres respecto a los hombres en los Servicios de Cirugía y Traumatología. Estas observaciones se deberían medir en los próximos años para analizar la evolución, elaborar hipótesis explicativas de lo sucedido y programar medidas de mejora de la calidad asistencial. En el análisis de la mortalidad respecto al sexo, comentar también que tres enfermedades, el IAM, la hernia abdominal y la infección de orina tienen una TM significativamente diferente entre sexos. El diseño de este estudio únicamente permite describir y no explicar las diferencias entre sexos, como el trabajo de la Sierra y cols., que analiza diferentes variables que pueden influir en la mortalidad (12). No obstante, en la literatura científica se ha descrito una mayor mortalidad de las mujeres con cardiopatía isquémica (13) y una mayor morbimortalidad de la infección urinaria en el varón respecto a las mujeres; como se recoge en este estudio (14).

Los servicios con mayor número de fallecimientos suelen ser los médicos, como en este caso, seguidos de los quirúrgi- 
TABLAV

DIAGNÓSTICO PRINCIPAL DE LOS FALLECIM IENTOS POR SEXO

\begin{tabular}{|c|c|c|c|c|c|c|c|}
\hline Código ClE-9-MC & Diagnóstico principal & $\begin{array}{l}\text { Hombres } \\
\text { № éxitus }\end{array}$ & $\begin{array}{c}\text { Hombres } \\
\text { TM } \\
\%\end{array}$ & IC $95 \%$ & $\begin{array}{l}\text { Mujeres } \\
\text { № éxitus }\end{array}$ & $\begin{array}{c}\text { M ujeres } \\
\text { TM } \\
\%\end{array}$ & IC $95 \%$ \\
\hline 38 & Septicemia & $5(55,6)$ & 29,4 & $11,4-56,0$ & $4(54,4)$ & 26,6 & $8,9-51,2$ \\
\hline-156 & plasia esófa & $16(69,6)$ & 8,5 & & $7(30,4)$ & 8,3 & 6,9 \\
\hline L55-157.9 & Neoplasia de hígado, vesícula, tracto biliar y páncreas & $9(18,2)$ & 16,7 & $8,4-29,8$ & $2(18,2)$ & 9,1 & $1,6-30,6$ \\
\hline $162,3-162,9$ & Neoplasia de bronquio y pulmón & $10(83,3)$ & 11,9 & $6,2-21,2$ & $2(16,7)$ & 18,2 & $3,2-52,2$ \\
\hline 185,188 & stata y vejiga & $8(100)$ & 6,1 & $2,9-12,1$ & $0(0)$ & 0 & $0-24,1$ \\
\hline $197,0-197,7$ & Neoplasia maligna secundaria de los & & & & & & \\
\hline aparatc & y digestivo & $6(50)$ & 25 & $10-47$ & $6(50)$ & 24 & $10,2-45,5$ \\
\hline 250 & Diabetes mellitus & $3(60)$ & 2,3 & $0,6-7,2$ & $2(40)$ & 2 & $0,3-7,7$ \\
\hline 410 & do de miocardio* & $15(45,5)$ & 7,7 & $4,5-12,5$ & $18(54,5)$ & 19,5 & $12,3-4,4$ \\
\hline 5,19 & epulmón & $7(70)$ & 25 & $11,4-45,2$ & $3(30)$ & 16,7 & $4,4-42,3$ \\
\hline $6,0,427$ & ntricular complet & $5(45,5)$ & 3 & $1,1-$ & $6(54,5)$ & 3,3 & $1,3-7,5$ \\
\hline 428 & Insufic & $9(34,6)$ & 8,7 & $4,3-16,3$ & $17(65,4)$ & 8,4 & $5,1-13,3$ \\
\hline $430-435.9$ & rovascular & $27(37)$ & 10,9 & $7,4-15,6$ & $33(63)$ & 13,6 & $9,7-18,8$ \\
\hline & Bron & $1(11,1)$ & 1,1 & 0,0 & $8(88,9)$ & 4,5 & $2,1-9,0$ \\
\hline 507.0 & nconeumonía y neumonía p & $30(57,7)$ & 9,7 & $6,8-13,8$ & $22(42,3)$ & 12,9 & $8,5-19,2$ \\
\hline 14 & nfisema y asma & $28(77,8)$ & 4,7 & 3,2 & $8(22,2)$ & 3,3 & $1,6-6,8$ \\
\hline $0-553$ & Hernia abdominal** & $2(25)$ & 0,6 & $(0,1$ & $6(75)$ & 4,3 & $1,8-9,6$ \\
\hline 557 & Insuficiencia & $6(35,3)$ & 60 & $27,3-86,3$ & $11(64,7)$ & 73,3 & $44,9-91,1$ \\
\hline 560 & stinal sin hernia & $6(54,5)$ & 13 & & & 14,3 & $5,4-31,0$ \\
\hline 571 & lad hepática crónica y cirrosis & $5(100)$ & 6,0 & 2,2 & $0(0)$ & 0 & $0-12,2$ \\
\hline $574-57$ & Colelitiasis, trastornos de la vesícula biliar y pancreatitis & $5(55,5)$ & 2,0 & $0,7-4,9$ & $4(44,5)$ & 1,2 & $0,3-3,2$ \\
\hline 578,9 & troduodenal y HGI & $7(53,8)$ & 4,6 & $2,0-9,6$ & $6(46,2)$ & 8,1 & $3,2-17,4$ \\
\hline $590.1,599,0$ & Pielonefritis aguda e infección urinaria, sitio no especificado*** & $5(100)$ & 4,9 & $1,8-11,6$ & $0(0)$ & 0 & $0-2,9$ \\
\hline & & $5(4$ & 100 & & $7(58,3)$ & 100 & $56,1-100$ \\
\hline & Fractura de cuello de fémur y pertrocant & & 10,6 & & $11(67,3)$ & 5,5 & $2,9-9,9$ \\
\hline
\end{tabular}

TM : tasa de mortalidad o letalidad. \% : porcentaje; IC 95\% : intervalo de confianza del 95\%. EPOC: enfermedad pulmonar obstructiva crónica, BCO: broncopatía crónica obstructiva. HGI: hemorragia gastrointestinal. Entre paréntesis porcentajes; * $p=0,005 ; * * p=0,01 ; * * * p=0,008$

cos y pediatría (7). En este caso el que mayor número de casos tuvo fue el de Medicina Interna, si bien el que mayor TM tuvo fue la Unidad de Cuidados Intensivos, como es lógico por la gravedad y peor pronósticos de los pacientes ingresados en dicha unidad.

En el estudio de las causas de los fallecimientos dentro del hospital se suele emplear para clasificar la enfermedades responsables del evento la CIE, ya sea agrupadas por códigos $(4,5)$, o por grandes grupos de enfermedades $(15,16)$. El análisis de la mortalidad según los GRD se ha empleado en menor número de ocasiones en la literatura nacional $(1,3,8)$. En este trabajo se analizó la mortalidad por diagnóstico principal según la CIE-9-MC y los GRD como en el estudio de García y cols. (3).

De forma general cuando se estudia la mortalidad por la clasificación de la CIE las principales causas son: cardiaca, respiratoria, digestiva y del sistema nervioso central (1,3$5,14,15)$. En este trabajo, las tres principales causas de muertes por GRD son las relacionadas con problemas cardiacos, respiratorios y vasculares del sistema nervioso; y si se clasifica los fallecimientos por el diagnóstico principal la causa es la patología pulmonar (neumonía más EPOC), seguida del problema vascular del sistema nervioso y patología cardiaca. No hay que olvidar las principales causas de mortalidad de los problemas quirúrgicos fueron la insuficiencia vascular del intestino (isquémia mesentérica) en Cirugía General y la fractura de fémur complicada en Traumatología.

Este trabajo tiene limitaciones como el no analizar todos los éxitus del hospital debido a que algunos de los fallecimientos llegan con retraso la Unidad de Documentación Clínica y por tanto no se han codificado a tiempo de realizar el trabajo, el porcentaje de historias revisadas en este trabajo $(88 \%)$ es similar al encontrado por otros autores $(86 \%)$ (5). Si bien aporta la TM y no únicamente los datos de mortalidad cruda como los trabajos de García y cols. (3) e incluye el IC $95 \%$ de los porcentajes, lo que pensamos da una información sobre el margen entre los que estará el porcentaje en la población general al no disponer del total de los éxitus.

La mortalidad hospitalaria es un indicador clásico de calidad asistencial. La obtención de los GRD y el diagnóstico principal de los éxitus es fácil a partir de la Unidad de Documentación. Por estos dos hechos aconsejamos la evaluación de la mortalidad de forma anual para así ver la tendencia en el tiempo y las posibles desviaciones, lo que permitiría emprender medidas de intervención sobre este indicador. Lo que a la larga redundaría en un mejor aprovechamiento de los recursos y en la calidad de la asistencia sanitaria. 


\section{Bibliografía}

1. García Ortega C, Almenara Barrios J, García Ortega JJ. Tasas específicas de mortalidad en el Hospital de Algeciras durante el periodo 19951996. Rev Esp Salud Pública 1997; 71: 305-315.

2. Segura Benedicto A. El análisis de la mortalidad hospitalaria como una medida de efectividad. Med Clin (Barc) 1988; 91: 139-141.

3. García C, Almenara J, Mérida FJ, Serrano P. Tasas de letalidad por GRD en un hospital general básico. Rev Calidad Asistencial 2000; 15 : 31-35.

4. Sánchez Bisono JR, Gómez-Rosich A, Amor Gea JF, García Sánchez MJ, Campoy Domene LF, Peña Migallón-Sánchez P. Análisis de la mortalidad hospitalaria en un hospital comarcal. An Med Interna (Madrid) 1997; 14: 71-75.

5. Ferrus Grau L. Estudio de mortalidad en un hospital comarcal. Todo Hospital 1996; 129: 53-58.

6. Longo DR, Bohr D. Métodos cuantitativos en la gestión de la calidad. Una guía práctiva. Barcelona: SG editores, 1994.

7. Rodríguez Navarro M, Griera Borrás JL, Asenjo Sebastián MA. Mortalidad Hospitalaria: análisis retrospectivo en el Hospital Clínico de Sevilla. Todo Hospital 1988; 53: 29-31.

8. Polo Ordoqui M, Arce Obieta JM. Variación en la tasa de letalidad en los APR-GRD con mayor frecuencia de fallecimientos durante los años 1992 y 1994 en la Fundación Jiménez Díaz. Gestión Hospitalaria 1995; 4: $20-22$.

9. Matorras Ga lán P, Alonso López F, Daroca Pérez R, Lamelas Olata JA, Díaz-Caneja N, Gancedo González Z. Mortalidad en Medicina Interna de un hospital de tercer nivel. Rev Clin Esp 1989; 185: 175-178.
10. Sicras Mainar A, Navarro Artieda R. Medida de la mortalidad como efecto de la intervención hospitalaria en un Servicio de Medicina Interna. An Med Interna (Madrid) 1992; 9: 21-29.

11. Escalante Yangüela B, Oncinis Torres R, Lacasa Marzo JJ, Candel Calderón M, Sanpedro Feliu JA. Estudio de mortalidad hospitalaria en el Servicio de Medicina Interna de una hospital comarcal. An Med Interna (Madrid) 1994; 11: 381-384.

12. Población española. Fuente: Instituto Nacional de Estadística. Anuario El País, 2000. Madrid: El País 2000.

13. De la Sierra A, Cardellach F, Sentis J, Bové A, Ingelmo M, UrbanoMárquez A. Estudio prospectivo sobre la mortalidad en un Servicio de Medicina Interna General. Med Clin (Barc) 1988; 91: 121-123.

14. Alcázar Quevedo M, Antón Pascual C, Bonet Pla A, Calabuig Pérez J, Jiménez Cruzado L, Navarro Pérez et al. Episodio de prevención de factores de riesgo cardiovascular por edad y sexo en la Comunidad Valenciana. Aten Primaria 1999; 23: 411-418.

15. Ronald AR, Harding GKM. Complicated urinary tract infections. Infec Dis Clin N Am 1997; 11: 583-592.

16. García Saavedra V. Benet Catala A, Pedro Silva P, Toda Savall R, Vidal Marsal F, Richart Jurado C. Estudio comparativo 1984-1989 de la mortalidad en el Servicio de Medicina Interna de un hospital general de segundo nivel. An Med Interna (Madrid) 1991; 8: 284-288.

17. Escolar Castellon F, Samperiz Legarre L, Pérez Poza A, Expósito Rando A, Merino Múñoz F, Castillo Parra C. Morbilidad, mortalidad y otros indicadores de la actividad asistencial en un Servicio de Medicina Interna General. An Med Interna (Madrid) 1993: 10: 327-332. 